\title{
Frequency Selectivity of a Normal and a Hybrid Bragg Resonators $^{*}$
}

\author{
Keiichi KAMADA, Yukihiro SOGA, Shuhei ODAWARA, Ken MISAWA, \\ Mitsuhiro YOSHIDA ${ }^{1)}$ and Naum S. GINZBURG ${ }^{2)}$ \\ Graduate School of Natural Science and Technology, Kanazawa University, Kakuma, Kanazawa 920-1192, Japan \\ ${ }^{1)}$ High Energy Accelerator Research Organization, KEK, 1-1 Oho, Tsukuba, Ibaraki 305-0801, Japan \\ ${ }^{2)}$ Institute of Applied Physics, Russian Academy of Science, Nizhny Novgorod 603600, Russia
}

(Received 7 December 2012 / Accepted 24 April 2013)

\begin{abstract}
A hybrid Bragg resonator with an advanced and a traditional Bragg reflectors was designed to develop the frequency selectivity of the free electron maser with frequency of $40 \mathrm{GHz}$ using an intense relativistic electron beam. A normal Bragg resonator with two traditional Bragg reflectors was also designed for the same frequency. The interaction between a propagating wave and a quasi-cutoff mode in an advanced Bragg reflector was expected to provide higher frequency selectivity than a traditional Bragg reflector. In the cold test, the frequency resolution of the hybrid Bragg resonator was appeared to be $\pm 0.05 \mathrm{GHz}$, while that of the normal one was $\pm 0.5 \mathrm{GHz}$.
\end{abstract}

(C) 2013 The Japan Society of Plasma Science and Nuclear Fusion Research

Keywords: free electron maser, bragg resonator, electron tube, intense electron beam, milimeter wave source

DOI: $10.1585 /$ pfr.8.2406154

\section{Introduction}

Solid state components, lasers and electron tubes are the candidates of compact light sources with the frequency range of $0.1-10 \mathrm{THz}(\mathrm{THz}$ source). $\mathrm{THz}$ sources using laser and solid state components with average output power of $\mu \mathrm{W}$ to $\mathrm{mW}$ have developed new tremendous possibilities of applications. Electron tubes are expected to realize steady state $\mathrm{THz}$ sources with output power of $\geqq$ $1 \mathrm{~W}$ or intense pulsed $\mathrm{THz}$ sources with power of $\gg 1 \mathrm{MW}$. The intense $\mathrm{THz}$ sources will be applied to thermonuclear fusion, particle accelerators, radars, etc. Among electron tubes, gyrotrons [1] with output power of $1 \mathrm{MW}$ and frequency of $170 \mathrm{GHz}$ have been ready for thermonuclear fusion.

A free electron maser (FEM) using an intense relativistic electron beam (REB) is one of the other candidates of intense $\mathrm{THz}$ sources. However, the FEM using REB shows the wide frequency spectrum of the radiation because of its strong self electric field. Usually, a normal Bragg resonator using two traditional Bragg reflectors is utilized to develop the frequency selectivity. In the cylindrical structure, the Bragg resonator is immersed in an axial guide and a helical wiggler magnetic fields. Traditional Bragg reflectors work as mirrors in the optical distributed feedback laser [2-4], as shown in Fig. 1. However, as the frequency increases, a traditional Bragg reflector loses the selective features over transverse indexes because of its oversized parameters. As a result, the radiation by an electron beam would represent an uncontrollable mixture of

author's e-mail: kkamada@staff.kanazawa-u.ac.jp

$\left.{ }^{*}\right)$ This article is based on the presentation at the 22 nd International Toki Conference (ITC22). the waveguide modes.

The problem described above can be partially solved by using the coupling between the propagating and the cutoff modes in an advanced Bragg structure [5,6]. A hybrid Bragg resonator utilizes an advanced Bragg reflector at the upstream side instead of a traditional one as shown in Fig. 1. The corrugation period for a traditional Bragg reflector can be determined by the relation $k=k_{\mathrm{b}} / 2$, Bragg condition, where $k$ is the axial propagation constant of the microwave to be reflected back to the circular waveg-

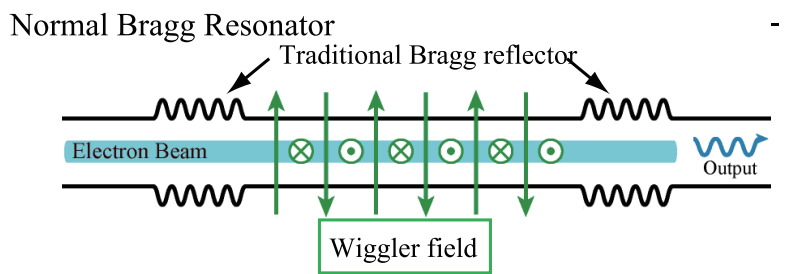

Hybrid Bragg Resonator

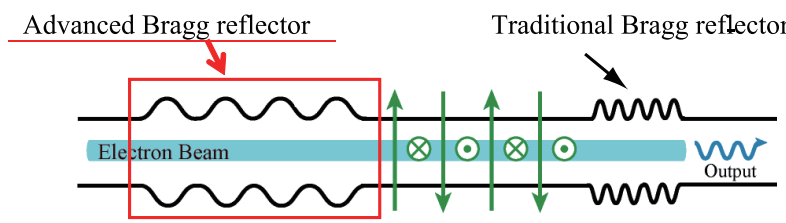

Fig. 1 Schematics of a normal (upper) and a hybrid (lower) Bragg resonators. An electron beam is injected from the left side. The corrugation period of an advanced Bragg reflector is longer nearly twice than that of a traditional Bragg reflector. 
uide and $k_{\mathrm{b}}=2 \pi / \lambda_{\mathrm{T}}$ and $\lambda_{\mathrm{T}}$ is the corrugation period. On the other hands, the corrugation period $\lambda_{\mathrm{A}}$ of an advanced Bragg reflector was nearly equal to $k \approx 2 \pi / \lambda_{\mathrm{A}}$, because the advanced Bragg reflector works like a resonator. Due to small variation of the mean radius of the advanced Bragg reflector at the boundaries, the quasi cutoff mode is trapped inside the advanced Bragg reflector.

Propagating waves interact with the beam in a circular waveguide located between two reflectors. A part of waves with wavenumber that satisfies the Bragg condition is reflected backward by a traditional Bragg reflector. Among the backward waves incident on the advanced Bragg reflector, only the wave coupled with the quasi cutoff mode is reflected to the downstream side and amplified by the electron beam. So that a hybrid Bragg resonator is expected to show higher frequency selectivity than a normal Bragg resonator.

Preliminary experimental results of the frequency response of the reflectors and resonators are reported in this paper.

\section{Design of Bragg Reflectors}

A traditional and an advanced Bragg reflectors of the cylindrical structure were designed by use of the mode coupling theory [7-9]. The calculated values of parameters of the Bragg reflectors were examined by the simulation code CST MW studio. The shape of corrugation of both reflectors is supposed to be sinusoidal. The design process is described below in order of a traditional and an advanced Bragg resonators.

In our case, the corrugation period, $\lambda_{\mathrm{T}}$, of the traditional Bragg reflector was calculated to be $3.9 \mathrm{~mm}$ for $40 \mathrm{GHz}, \mathrm{TE}_{11}$ mode of the circular waveguide with the mean radius of $R=8.4 \mathrm{~mm}$.

To obtain narrower frequency spectrum and higher reflectance, the corrugation amplitude, $H$, was decided to be $0.25 \mathrm{~mm}$ from the calculated result shown in Fig. 2 where the axial length of the reflector is $400 \mathrm{~mm}$. The larger corrugation amplitude shows the higher reflectance with wider spectrum. As the corrugation amplitude increased, the more robust reflection of the incident wave by each corrugation was established but the effective optical path difference became more ambiguous.

The axial length, $L$, of the reflector was decided to be $250 \mathrm{~mm}$ from the calculated result of the dependency of the reflectance on the axial length of the traditional Bragg reflector, as shown in Fig. 3. As the axial length of the reflector increases, the reflectance increases. The reflected wave detected outside the Bragg reflector is superimposed by small reflected waves by each corrugation. The increase of the axial length represents the increase of the number of the reflected waves by each corrugation.

The designed parameters of the traditional Bragg reflector are described in Table 1. The calculated result by use of the mode coupling theory showed good agree-

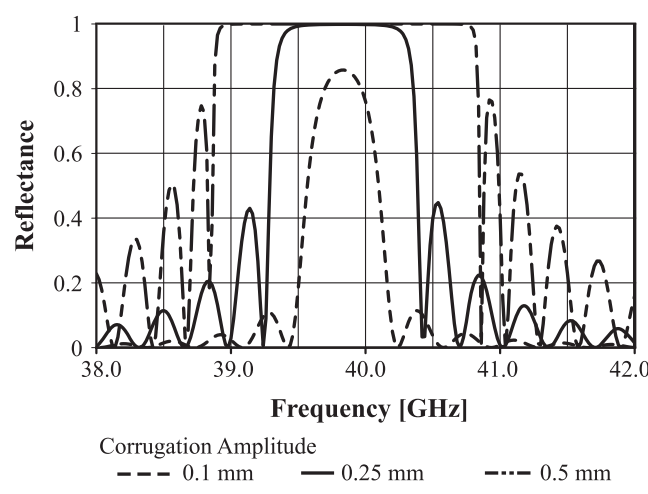

Fig. 2 The reflective spectra with different corrugation amplitudes of the traditional Bragg reflector calculated from a mode coupling theory where $L=400 \mathrm{~m}, \lambda_{\mathrm{T}}=3.9 \mathrm{~mm}$ and $R=8.4 \mathrm{~mm}$.

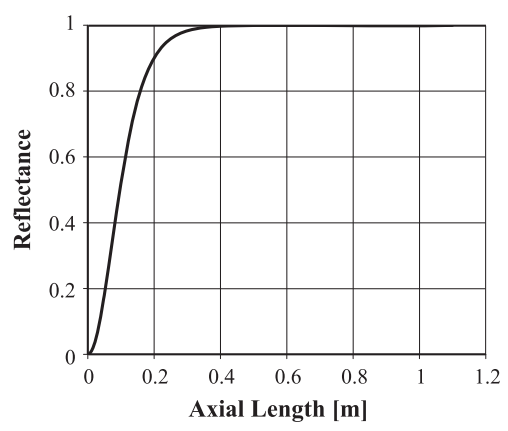

Fig. 3 The dependency of the reflectance on the axial length of the traditional Bragg reflector calculated from a mode coupling theory with $\lambda_{\mathrm{T}}=3.9 \mathrm{~mm}$, corrugation amplitude, $H=0.25 \mathrm{~mm}$, and $R=8.4 \mathrm{~mm}$.

Table 1 Parameters.

\begin{tabular}{|c|c|c|}
\hline & Traditional & Advanced \\
\hline mode & $\mathrm{TE}_{11}$ & $\mathrm{TE}_{11} \rightleftharpoons \mathrm{TM}_{12}$ \\
\hline Center Frequency [GHz] & 39.8 & 39.8 \\
\hline Resolution [GHz] & 0.5 & 0.05 \\
\hline Mean Radius [mm] & 8.4 & 8.4 \\
\hline Corrugation Period [mm] & 3.9 & 7.6 \\
\hline Corrugation Amplitude [mm] & 0.25 & 0.1 \\
\hline Corrugation Length [mm] & 250 & 200 \\
\hline
\end{tabular}

ment with the simulated one with parameters on Table 1 as shown in Fig. 4.

We applied mainly the simulated results to set the structural parameters of an advanced Bragg reflector. For example, the corrugation amplitude affects on the width and the center frequency of the reflective spectrum as shown in Fig. 5. After we tried many combinations of parameters, the corrugation period, $\lambda_{\mathrm{A}}$, was decided to be $7.6 \mathrm{~mm}$, the axial length was set to $200 \mathrm{~mm}$ and the corrugation amplitude was $0.1 \mathrm{~mm}$. The reflective spectrum of the advanced Bragg reflector with parameters in Table 1 is also shown in Fig. 4. The advanced Bragg reflector shows 


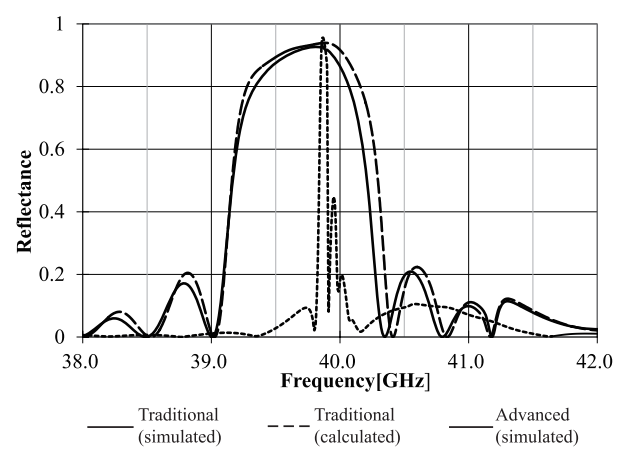

Fig. 4 Calculated result (dashed line) based on the mode coupling theory and simulated result (solid line) for the traditional Bragg reflector show good agreement. The simulated result of the advanced Bragg reflector (dotted line) is also shown. Parameters of the reflectors are shown in Table 1.

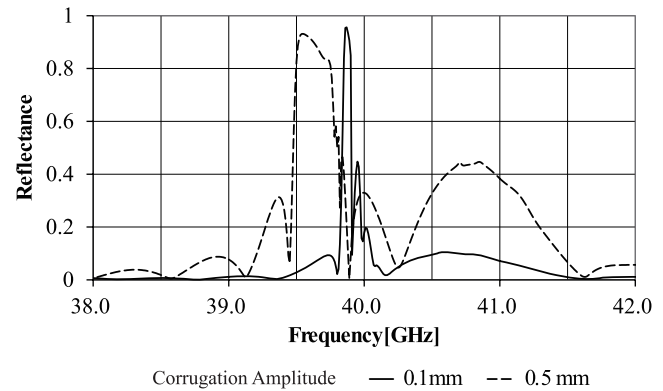

Fig. 5 The reflective spectra with two different corrugation amplitudes of the advanced Bragg reflector with $L=$ $200 \mathrm{~mm}, \lambda_{\mathrm{A}}=7.6 \mathrm{~mm}$ and $R=8.4 \mathrm{~mm}$.

narrower reflective spectrum around the center frequency than the traditional Bragg reflector. The center frequency of $39.8 \mathrm{GHz}$ is nearly equal to the cut off frequency of the $\mathrm{TM}_{12}$ mode of the advanced Bragg reflector. It is suggested that the coupling between the propagating $\left(\mathrm{TE}_{11}\right)$ and the quasi cut off mode $\left(\mathrm{TM}_{12}\right)$ in the advanced Bragg reflector operated as expected and the reflected microwave of $\mathrm{TE}_{11}$ mode was observed.

\section{Experimental Results and Discus- sions}

The traditional and the advanced Bragg reflectors with sinusoidal corrugation were made. The network analyzer E8354C (Agilent Technology) was utilized to carry out the cold tests of the Bragg reflectors and resonators.

In the cold test of both Bragg reflectors, the microwave was input by a horn from one side of the Bragg reflector and that passed through the reflector was detected by a horn antenna at another side.

The transmission spectra of the traditional and the advanced Bragg reflector obtained by cold tests are shown in Fig. 6. The simulated reflective spectra of the Bragg reflectors are also shown in Fig. 6. The experimental results

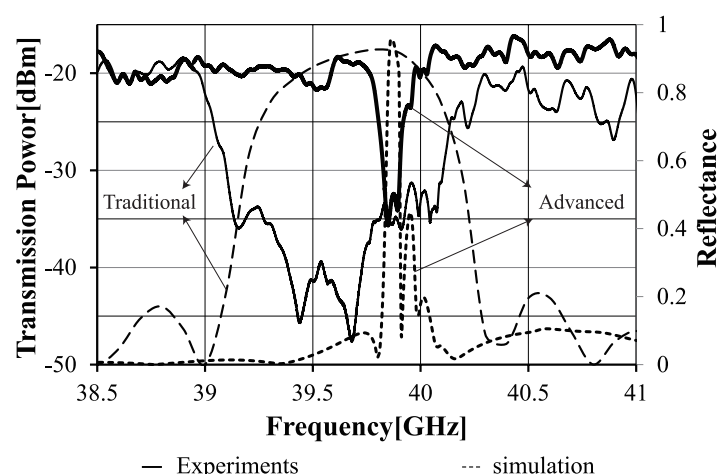

Fig. 6 Experimental results of transmission spectra (solid line) and simulated results of reflective spectra (dotted line) of the traditional and the advanced Bragg resonators.

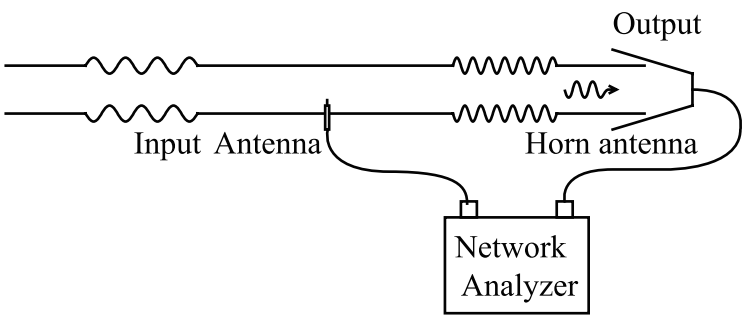

Fig. 7 Schematics of the experimental setup of cold test of the Bragg resonators. Microwave was input by an antenna inserted in the circular waveguide at the center of the axial length. The microwave passed through the traditional Bragg resonator was detected by a horn antenna.

show good agreements with the simulated results in their frequency range. As the width of the transmission spectrum around the center frequency of the advanced Bragg reflector is about 10 times narrower than that of the traditional one, the advanced Bragg reflector could work as expected.

In the cold tests of the normal and the hybrid Bragg resonators, microwave was input by a dipole antenna at the center of the circular waveguide located between Bragg reflectors as shown in Fig. 7. And the microwave passed through the traditional Bragg reflector located at the downstream side of both resonators was detected by a horn antenna.

The distance of the two reflectors, i.e. the length of the circular waveguide between the reflectors, was changed from 100 to $500 \mathrm{~mm}$ with $100 \mathrm{~mm}$ increments in between. It affected the transmission spectra of both resonators. The length was not optimized yet in both resonators.

The experimental result of the normal Bragg resonator with the distance of the two reflectors of $500 \mathrm{~mm}$ is shown in Fig. 8. Several peaks were found in the range of $39.6 \pm 0.5 \mathrm{GHz}$ approximately. The frequencies of the peaks were slightly changed and the number of peaks increased as the distance of the reflectors increased. In any length, the frequency range in which the peaks were ob- 


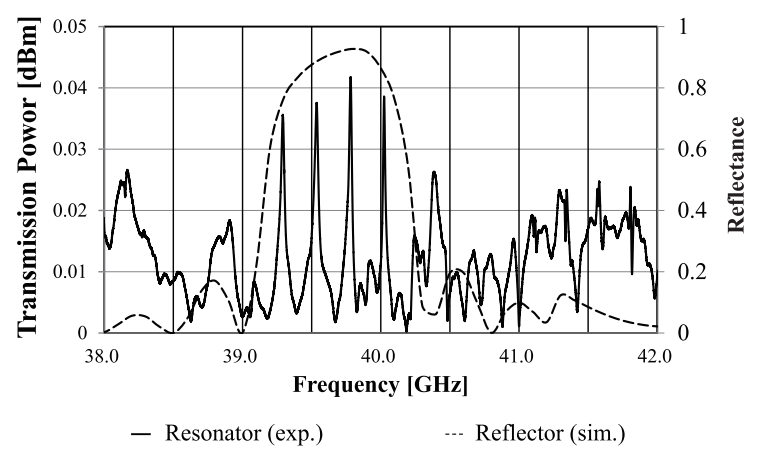

Fig. 8 Transmission spectrum obtained by the cold test (solid line) of the normal Bragg resonator and simulated reflective spectrum (dotted line) of the traditional Bragg reflector. The distance between reflectors is $500 \mathrm{~mm}$. Several peaks were observed in the range of $39.6 \pm 0.5 \mathrm{GHz}$ approximately. The range of the peaks is within the width of the simulated reflective spectrum of the traditional Bragg reflector.

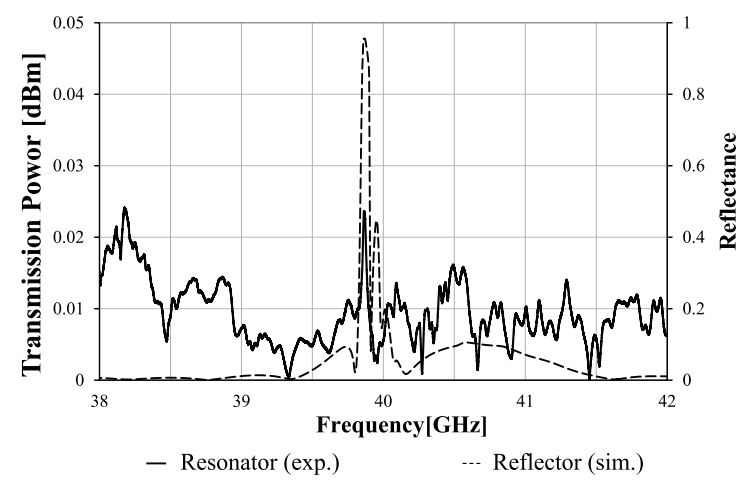

Fig. 9 Transmission spectrum obtained by the cold test (solid line) of the hybrid Bragg resonator and simulated reflective spectrum (dotted line) of the advanced Bragg reflector. The distance between reflectors is $500 \mathrm{~mm}$. The width of the peak frequency is $39.85 \pm 0.05 \mathrm{GHz}$.

served was within the width of the simulated reflective spectrum of the traditional Bragg reflector.

The frequency spectrum passed through the hybrid Bragg resonator with the distance of the two reflectors of $500 \mathrm{~mm}$ is shown in Fig. 9. A single narrow peak is observed at the same frequency of the advanced Bragg reflector. As the distance of the two reflectors increased, the frequency width of the peak decreased but the number of the peak was kept one. The output power is lower than the normal Bragg resonator. Though the distance of two reflectors was changed with $100 \mathrm{~mm}$ increments in between in the experiments, it should be changed precisely to find the appropriate distance.

While several peaks were observed with frequency range of about $39.6 \pm 0.5 \mathrm{GHz}$ using the normal Bragg resonator (Fig. 8), a single peak was obtained with the width about $39.85 \pm 0.05 \mathrm{GHz}$ using the hybrid Bragg resonator (Fig. 9). The hybrid Bragg resonator shows higher frequency selectivity than the normal one as expected. However, the problem of the lower output of the hybrid Bragg resonator than the normal one must be solved.

\section{Summary}

The results of the normal and the hybrid Bragg resonator derived from the mode coupling theory showed good agreements with the results of the cold experiments. The advanced Bragg reflector showed narrower frequency spectrum around the designed center frequency than the traditional one. As a result, the hybrid Bragg resonator presented a single narrow frequency spectrum.

\section{Acknowledgement}

The authors gratefully acknowledge Mr. Fumichika Kondo, Mr. Kota Nose, Miss Shoko Yanagi, Mr. Taro Kitamura, Mr. Naohisa Yamada, Mr. Kazuma Iwata, Mr. Kiyotaka Yasuda and Mr. Tomoaki Ogawa for their helpful assistance.

[1] A. Kasugai, K. Sakamoto, K. Takahashi, K. Kajiwara and N. Kobayashi, Nucl. Fusion 48, 054009 (2008).

[2] G.G. Denisov and M.G. Reznikov, Radiophys. Quantum Electron. 48, 407 (1982).

[3] I. Boscolo, Appl. Phys. B57, 217 (1993).

[4] T.S. Chu, F.V. Hartemann, B.G. Danly and R.J. Temkin, Phys. Rev. Lett. 72, 2391 (1994).

[5] N.S. Ginzburg, A.M. Malkin, N. Yu. Peskov, A.S. Sergeev, A.K. Kaminsky, S.N. Sedykh, E.A. Perelshtein, A.P. Sergeev and A.V. Elzhov, Phys. Rev. ST-AB 8, 040705 (2005).

[6] N.S. Ginzburg, A.M. Malkin, N. Yu. Peskov, A.S. Sergeev, V. Yu. Zaslavsky, K. Kamada and Y. Soga, Appl. Phys. Lett. 95, 043504 (2009).

[7] R.B. Mccowan, Int. J. Electron. 65, 463 (1988).

[8] C.K. Chong, D.B. McDermott, M.M. Razeghi, N.C. Luhman, Jr., J. Pretterexner, D. Wgner, M. Thummm, M. Caplan and H. Hullke, IEEE Trans. Plasma Sci. 20, 393 (1992).

[9] V.L. Bratman, G.G. Denisov, N.S. Ginzburg and M.I. Petelin, IEEE Trans. Quantum Electron. 19, 282 (1983). 